\title{
Percutaneous Injuries and Transmission of HIV Among Cases Referred for Post Exposure Prophylaxis to Razi Hospital in Ahvaz, a City in the Southwest Iran
}

\author{
Seyed Mohammad Alavi ${ }^{1,}$, Mehrdad Sharifi $^{2}$ \\ ${ }^{1}$ Health Research Institute, Jundishapur Infectious and Tropical Diseases Research Center, Ahvaz Jundishapur University of Medical Sciences, Ahvaz, IR Iran \\ ${ }^{2}$ Health Expert of Ahvaz health Center, Ahvaz Jundishapur University of Medical Sciences, Ahvaz, IR Iran \\ ${ }^{*}$ Corresponding author: Seyed Mohammad Alavi, Jundishapur Infectious and Tropical Diseases Research Center, Infectious Disease Ward of Razi Hospital, Ahvaz, IR Iran. Tel: +98- \\ 6113387724, Fax:+989161184916, E-mail: alavi.seyedmohammad@yahoo.com.
}

Received: September 21, 2012; Revised: January 11, 2013; Accepted: January 23, 2013

\begin{abstract}
Background:Health care workers(HCWs) and public in Iran may be at the risk of percutaneous injury(PI) as well as infection with human immunodeficiency virus (HIV).

Objectives: The aim of this study was to evaluate the PI and transmission of HIV infection in PI cases referred to an HIV preventive focal point unit.

Patients and Methods: In this prospective study,369 PI cases who had referred to Razi Hospital of Ahvaz, from 1993 to 2008 were studied. PI cases were placed in three groups: occupational (HCWs), accidental (children) and victims of a crime (women). The data including demographic, occupational, PI incidence place, post-exposure prophylaxis (PEP) and follow-up tests for HIV infection were analyzed in SPSS 16 using chi square test.

Results: From a total of 369 cases, 273 (74\%) were HCWs, among which nurses (70\%) were the most involved employees, followed by surgeons (18.3\%) and lab workers (8.4\%). Fifty five cases (14.9\%) were accidental needle stick (NS) and 41 (11.1\%) were victims. Among HCWs, 38 (13.8\%) were injured by needles soiled with HIV infected bloods. Only 105 (28.4\%) needed PEP, among which 92 (87.6\%), including 50 surgeons, 21 nurses and 18 lab workers were HCW. The results of HIV tests in all cases were negative.

Conclusions: Although nurses are the most involved occupational group in documented NS, surgeons are the most important high risk HCW who need PEP. No case of seroconversion has taken place as a result of PI.This means that the current PEP may have an effective role in HIV prevention.
\end{abstract}

Keywords: Percutaneous Injury; Needle Stick; Health Care Worker; HIV Infection; Nosocomial Infection

\section{Background}

Accidental exposure to biological materials has been attracting an increasing attention in the recent years due to the probability of serious infections and consequent dangerous health damages. Blood borne infections (BBIs) are major concerns for all healthcare workers (HCWs). Percutaneous injury (PI) by sharp instruments such as needle stick injuries (NIs) are one of the potential modes for transmission of such infections. In USA, it is estimated that 600000 to 800000 sharp injuries (SI) occur each year among HCWs, while in the UK it is 100000 injuries per year (1). All HCWs performing invasive procedures with sharp instruments are at the risk of injury (2). These injuries can result in substantial health consequences as well as psychological stress for HCWs and their families. Occupational transmission of HIV among HCW is well documented (3-9).
Downstream risks in the cleaning personnel, who are at a high risks, especially in emerging countries with lots of care procedures but suboptimal waste management, are a subject of interest. In a national case surveillance study during 20 years of HIV epidemy in the United States, 57 cases of occupational HIV infection were registered, while 24 (42\%) of them occurred in nurses (3). A guideline called standard precaution, aimed at preventing the accidental exposure to blood borne pathogens recommended by CDC in 1996 (5). Standard precautions are the control practices, used to prevent the transmission of blood/ fluid borne infections via non-intact skins and mucous membranes. These measures are used when providing care to all individuals.

According to the health policy of Iranian ministry of health, all accidental injuries, exposed to the blood borne pathogens especially HIV, are advised to get evaluated in order to prevent the HIV infection. A selected hospital and

Implication for health policy/practice/research/medical education:

The results of this study are useful for health policies of HIV control. The results can be used by general physicians and other health care workers in preventive fields of health.

Copyright (C) 2013, Ahvaz JundishapurUniversity of Medical Sciences; Published by Kowsar Corp. This is an open-access article distributed under the terms of the Creative Commons Attribution License, which permits unrestricted use, distribution, and reproduction in any medium, provided the original work is properly cited. 
a selected infectious disease sub-specialist (defined as HIV/AIDS focal point) have been appointed by the health center. All accidental cases suspected to be exposed to HIV have been referred to these focal points (6).

\section{Objectives}

Considering the lack of epidemiological studies on accidental NS and HIV transmission caused by percutaneous injuries in Ahvaz, the present study was conducted. The aim was to evaluate the NS injuries and identify the risk of HIV infection in the NS cases referred to a teaching hospital.

\section{Patients and Methods}

Study design: prospective surveillance study. Place of study: HIV/AIDS focal point unit.

Participants: three hundred and sixty nine cases including HCW, children and women referred for PI to the Razi Hospital, a teaching hospital affiliated to Jundishapur University of Medical Sciences in Ahvaz, capital of Khuzestan province, southwest Iran, from 1993 to 2008.

HCWs were registered as occupational NS/SI; children were registered as accidental NS and young women as victims of a criminal NS. Children were injured when playing with bared feet in parks, where used injecting equipments were found. Women were injured by a needle in the streets where they had been attacked by a stray young man suspected to be an injecting drug user (IDU).

According to the standard precautions recommended by Iranian $\mathrm{CDC}$, cases have been categorized in three groups (6) as: high risk, intermediate and low risk exposure.

Individuals have been defined as high risk if: exposure occurred within the last 72 hours, injury had been occurred by a hallow needle soiled with blood of a source person which was HIV-positive or the HIV status was unknown but with high risk behavior (e.g. IDU).

Individuals have been defined as low risk if: the source person was HIV negative.

Individuals have been defined as intermediate if: the event was occurred in a condition between the abovementioned situations.

Post-exposure prophylaxis (PEP) had been given to HCWs injured by seropositive sources (the high risk group), the intermediated group had been evaluated and if indicated had received PEP, but no drugs had been prescribed for the low risk group (6). Follow-up had been done after three and six months of exposure $(6,10)$.

Participants included all referred PIs (NS/SI) during the mentioned time period and no calculation was done for the sample size. Demographic and occupational data as well as the information about the PI incidence place, kind of injury, previous injuries, health education, post-exposure prophylaxis (PEP) and follow-up tests for HIV infection were derived out and recorded.
Statistical analysis: the acquired data of cases who received PEP were compared with those without PEP via SPSS for Windows (version 16; SPSS Inc., USA) using Student's t-test to compare the mean values in addition to the chi square test. Results were regarded as significant when $\mathrm{P}<0.05$.

\section{Results}

From a total of 369 referred cases, 232 (62.9\%) were female. The mean age was $32.7 \pm 20.5$ years (ranging $2-59$ ). Two hundred and seventy three cases (74\%) were HCWs, among which nurses with the frequency of 191 (70\%) were the most involved HCWs, followed by 50 (18.3\%) surgeon and $23(8.4 \%)$ lab workers (Table 1). Fifty five cases (14.9\%) had accidental NS (ranging 2 - 15 years) and 41 (11.1\%) were victims of criminal NS (women) with an age range of 15 40 years. Among HCWs, 38 (13.8\%) were injured by needles soiled with HIV infected bloods.

Table 1. Distribution of Occupational Needle Stick/Sharp Injury Among Health Care Workers in the Hospital Departments

\begin{tabular}{|c|c|c|}
\hline $\mathbf{H C W}^{\mathrm{a}}$ & Department & No. (\%) \\
\hline \multicolumn{3}{|c|}{ Nurses $(n=191)$} \\
\hline & Emergency & $80(29.3)$ \\
\hline & Operating room & $72(26.4)$ \\
\hline & Infectious diseases & $13(4.8)$ \\
\hline & Internal medicine & $10(3.7)$ \\
\hline & Orthopedy & $8(2.9)$ \\
\hline & $\mathrm{ICU}^{\mathrm{a}}$ & $8(2.9)$ \\
\hline \multicolumn{3}{|c|}{ Practitioners $(n=55)$} \\
\hline & General surgery & $28(10.3)$ \\
\hline & Orthopedic surgery & $22(8.1)$ \\
\hline & Anesthesiology & $4(1.5)$ \\
\hline & Gasterointestine & $1(0.3)$ \\
\hline \multicolumn{3}{|c|}{ Lab personnel $(n=27)$} \\
\hline & Workers & $23(8.4)$ \\
\hline & Expert & $2(0.7)$ \\
\hline & Doctors & $2(0.7)$ \\
\hline
\end{tabular}

a Abbreviations: HCW, Health care worker; ICU: Intensive care unit.

From a total of 369 , only 105 cases (28.4\%) needed preventive therapy with anti-retroviral drugs, among which 92 (87.6\%) cases, including 50 surgeons, 21 nurses and 18 lab workers had occupational NS. Five $(4.8 \%)$ had accidental and 8 (7.6\%) criminal NS. Only 105 (28.4\%) needed PEP, among which 92 (87.6\%), including 50 surgeons, 21 nurses and 18 lab workers were HCW. The results of HIV tests in all cases were negative.

The results of HIV tests performed for all cases were negative. None of the cases converted from negative to positive results. There were no significant differences ( $P$ 
Alavi SM et al.

$>0.05$ ) in the defined variables, except the kind of exposure, between those who received antiretroviral drugs and those who received no drugs (Table 2).

Table 2. Comparison Between Percutaneous Injured Cases Who Received Post-Exposure Prophylaxis and Those Who Received no Drugs

\begin{tabular}{|c|c|c|c|}
\hline Variables & PI with PEP, No. (\%), n= 105 & PI without PEP, $n=264$, No. (\%) & PValue \\
\hline Age, mean (SD), y & $27.3 \pm 18.1$ & $23.8 \pm 19.2$ & 0.10 \\
\hline \multicolumn{4}{|l|}{ Sex } \\
\hline Female & $70(66.7)$ & $158(60)$ & 0.23 \\
\hline Male & $35(33.3)$ & $106(40)$ & \\
\hline \multicolumn{4}{|l|}{ Health education } \\
\hline Educated & $95(90.5)$ & $229(86.7)$ & 0.38 \\
\hline Previous injury & $13(12.4)$ & $26(9.8)$ & 0.45 \\
\hline \multicolumn{4}{|c|}{ Kind of exposure, needle soiled with: } \\
\hline HIV $^{\mathrm{a}}+$ blood & $38(36.2)$ & $0(0.0)$ & $<0.0001$ \\
\hline IDUs blood & $55(52.3)$ & $103(30.9)$ & 0.01 \\
\hline Unknown blood & $12(11.5)$ & $161(69.1)$ & $<0.0001$ \\
\hline
\end{tabular}

a Abbreviations: HIV, human immunodeficiency virus; IDU, injecting drug user; PI, percutaneous injured; PEP, post-exposure prophylaxis.

\section{Discussion}

The results of this study revealed that nurses are the most involved occupational group (29.3\%) in the documented NS, followed by surgeons representing $18.4 \%$ of the occupational injuries referrals who are exposed to the biological materials such as blood and are at high risk of infection with blood borne pathogens such as HIV. This finding is consistent with other studies (10-12).

The present study also showed that surgeons were the most frequent at-risk group of HCWs with 54.3\% PEP needed exposure. Perry et al. showed that the highest rate of occupational injuries was among surgical assistants, closely followed by nurses (13). In another study of blood and body fluid exposure among hospital physicians in the United Kingdom, 46\% of blood and body fluid exposures during a course of three years, occurred in senior physicians and 54\% in junior physicians (14). It has been extensively studied that increased risk of exposure is associated with some factors such as type of surgery, time of procedure, whether a procedure that involved a needle that has been placed in the patient's source vein or artery, kind of used device in the surgery and experience of the surgeon $(3,4,6-10)$. This study also showed that the majority of needle stick injuries especially those accidentally occurred out of the hospitals are minor injuries and should not be considered as PEP candidates.

Emergency room and operating room with the respective frequency of $29.3 \%$ and $26.4 \%$ for the nurses as well as operation room with $19.9 \%$ for the surgeons were the most important hospital departments for occupational NS/SI. Myers et al. in a related analysis showed that 6.4 sharp device-related blood and body fluid exposures per 1,000 surgical procedures have occurred in the operating rooms (15). We think that the risk of exposure is directly correlated with the estimated amount of blood lost by the patient during a procedure, the number of injections, and the number of personnel working in the emergency or surgical fields. Although the nursing professionals are trained in the standard precautions during their education and admission in tertiary hospitals, this information indicates that the employed strategies should be reviewed for future training, emphasizing on the avoidance of PI.

The present study also showed that surgeons were the most frequent group of at-risk HCWs with 54.3\% PEP needed exposure. Perry et al. showed that the highest rate of occupational injuries were among surgical assistants, closely followed by nurses (13). In another study of blood and body fluid exposure among hospital physicians in the United Kingdom, $46 \%$ of blood and body fluid exposures during a course of three years occurred in senior physicians and 54\% in junior physicians (14). It has been extensively studied that increased risk of exposure is associated with some factors such as kind of surgery, time of procedure, whether a procedure that involved a needle was placed in the patient's source vein or artery, kind of used device in the surgery and experience of the surgeon (3, 4, 6-10).

This study also showed that the majority of needle stick injuries especially those accidentally occurred out of the hospitals are minor injuries and should not be considered as PEP candidates. From the total of 96 people in the present study who had accidental or crime needle stick 
injuries, risk assessment showed that only about $12 \%$ of them required PEP. Since most of these exposures take place upon clothing or shoes and the HIV positivity of the causing needle is in doubt, they do not need PEP. This finding is in agreement with the fact about HIV transmission in the literature (16). We found no similar study in this issue to compare with our results.

In the present study, no case of HIV infection occurred in all cases of accidental injuries registered in the hospital during 15 years. Previous studies in the world indicate that HIV infection as a consequent outcome of occupational accident with biological material is a documented medical problem $(3,5,7,9,16)$. Over all, the risk of infection via needle stick is approximately $0.3 \%$ (16). Our finding is in consistent with the studied performed in India (10) and Algeria (17), In which no case of seroconversion has taken place so far as a result of needle stick injuries. In discussion, we have no clear reason for this difference between our finding and the results of other researches. We believe that some factors might be involved this situation e.g. 1) possibility of very effective preventive protocol for needle stick injuries employed in our hospital, 2) over-diagnosed high risk injuries resulting in PEP, 3) alertness of our HCWs and other injured individuals in early referring to the focal point hospitals during the golden time (in the first hours after injury), 4) low HIV infection prevalence in our country, and 5) small number of accidental NS in our study.

This study has several potential limitations, primarily because it was a retrospective review of the existing data obtained from one hospital and the number of NS case were relatively small. Report bias may have occured if the health care workers preferentially reported the exposure that they believed was more likely to result in HIV transmission or for which they wanted PEP (or both).

This study showed that although nurses are the most involved occupational group in the documented NS, surgeons are the major high-risk HCWs who need PEP. No case of seroconversion has taken place as a result of PI. This means that the current PEP may have an effective role in HIV prevention.

\section{Acknowledgements}

The authors wish to thank the personnel of Khuzestan health center especially Dr. Eisazadeh, Mr. Taei and archivist of HIV preventive unit in Razi hospital (focal point) for their kind cooperation.

\section{Authors' Contribution}

None declared.

\section{Financial Disclosure}

There is no interest to disclose.

\section{Funding/Support}

There was no funding or Support.

\section{References}

1. Trim JC, Elliott TSJ. A review of sharps injuries and preventative strategies. J Hospital Infect. 2003;53(4):237-242.

2. Prüss-Üstün Annette, Rapiti Elisabetta, Hutin Yvan. Estimation of the global burden of disease attributable to contaminated sharps injuries among health-care workers. Am J Industrial Med. 2005;48(6):482-490.

3. Do Ann N, Ciesielski Carol A, Metler Russ P, Hammett Teresa A Li Jianmin, Fleming Patricia L. Occupationally Acquired Human Immunodeficiency Virus (HIV) Infection: National Case Surveillance Data During 20 Years of the HIV Epidemic in the United States. Infect Cont Hospital Epidemiol. 2003;24(2):86-96.

4. Elmiyeh B, Whitaker IS, James MJ, Chahal CAA, Galea A, Alshafi K. Needle-stick injuries in the National Health Service: a culture of silence. JRSM. 2004;97(7):326-327.

5. Garner Julia S. Guideline for Isolation Precautions in Hospitals. Infect Cont Hospital Epidemiol. 1996;17(1):53-80.

6. [Report of HIV/AIDS] . Center of Control of Diseases, Ministry of Health of Iran. 2004.

7. Ippolito Giuseppe, Puro Vincenzo, Heptonstall Julia, Jagger Janine, De Carli Gabriella, Petrosillo Nicola. Occupational Human Immunodeficiency Virus Infection in Health Care Workers: Worldwide Cases Through September 1997. Clin Infect Dis. 1999;28(2):365-383.

8. Rapparini Cristiane. Occupational HIV infection among health care workers exposed to blood and body fluids in Brazil. Am J Infect Cont. 2006;34(4):237-240.

9. Tarantola A, Abiteboul D, Rachline A. Infection risks following accidental exposure to blood or body fluids in health care workers: A review of pathogens transmitted in published cases. Am J Infect Con. 2006;34(6):367-375.

10. Mehta A, Rodrigues C, Singhal T, Lopes N, D`Souza N, Sathe K, et al. Interventions to reduce needle stick injuries at a tertiary care centre. Indian J Med Microbiol. 2010;28(1):17.

11. Clarke Sean P, Rockett Joan L, Sloane Douglas M, Aiken Linda H Organizational climate, staffing, and safety equipment as predictors of needlestick injuries and near-misses in hospital nurses. Am J Infect Cont. 2002;30(4):207-216.

12. Trinkoff Alison M, Le Rong, Geiger-Brown Jeanne, Lipscomb Jane. Work Schedule, Needle Use, and Needlestick Injuries Among Registered Nurses. Infect Cont Hospital Epidemiol. 2007;28(2):156-164.

13. Perry Jane, Parker G, Jagger Janine. Scalpel blades: reducing in jury risk. Adv Exp Prevent. 2003;6(4):37-48.

14. Naghavi SHR, Sanati KA. Accidental blood and body fluid exposure among doctors. Occupational Med. 2009;59(2):101-106.

15. Myers Douglas J, Epling Carol, Dement John, Hunt Debra. Risk of Sharp Device-Related Blood and Body Fluid Exposure in Operating Rooms. Infect Cont Hospital Epidemiol. 2008;29(12):1139-1148.

16. Rio CD, Curran JW. Epidemiology and prevention of acquired im munodeficiency syndrome and human immunodeficiency virus infection. 7 ed. Mandell GL Bennett JE, Dolin R editor. : Philadelphia, Churchill Livingston,; 2010.

17. Beghdadli B, Ghomari O, Taleb M, Belhaj Z, Belabed A, Kandouci del AK, et al. [Personnel at risk for occupational blood exposure in a university hospital in West Algeria]. Sante Publique. 2009;21(3):253-61. 